\title{
Role of nanotechnology in diagnosing and treating COVID-19 during the Pandemic
}

\section{Abdul Waris ${ }^{1}$, Muhammad Ali ${ }^{1}$, Atta Ullah Khan², Asmat Ali ${ }^{3}$ and Abdul Baset ${ }^{4 *}$}

\author{
1Department of Biotechnology, Quaid-i-Azam University, Islamabad, Pakistan \\ 2Department of Biotechnology, University of Malakand, Chakdara dir Lower, Pakistan \\ ${ }^{3}$ Centre for Human Genetics, Hazara University Mansehra, Pakistan \\ ${ }^{4}$ Department of Zoology, Bacha Khan University Charsadda, Pakistan
}

\section{Abstract}

The coronavirus disease 2019 (COVID-19) pandemic, caused by the severe acute respiratory syndrome coronavirus 2 (SARS-CoV-2), began in December 2019 in Wuhan, China. To date, the virus has infected roughly 5,000,000 people and caused approximately 345,000 deaths worldwide, and these numbers are increasing rapidly. Because of the rapid spread and the rising disease burden, several antiviral drugs and immunomodulators are in clinical trials, but no drugs or vaccines have yet been approved against this deadly pandemic. At present, computed tomography scanning and reverse transcription (RT)-PCR are used to diagnose COVID-19, and nanotechnology is being used to develop drugs against COVID-19. Nanotechnology also plays a role in diagnosing COVID-19. In this article, we discuss the role of nanotechnology in diagnosing and potentially treating COVID-19.

\section{More Information}

*Address for Correspondence: Abdul Baset, Department of Zoology, Bacha Khan University Charsadda, Pakistan,

Email: drabdulbaset@bkuc.edu.pk

Submitted: 06 May 2020

Approved: 26 May 2020

Published: 27 May 2020

How to cite this article: Waris A, Ali M, Khan $\mathrm{AU}$, Ali A, Baset A. Role of nanotechnology in diagnosing and treating COVID-19 during the Pandemic. Int J Clin Virol. 2020; 4: 065-070.

DOI: 10.29328/journal.ijcv.1001017

Copyright: @ 2020 Waris A, et al. This is an open access article distributed under the Creative Commons Attribution License, which permits unrestricted use, distribution, and reproduction in any medium, provided the original work is properly cited.

Keywords: COVID-19; SARS-CoV-2; Nanotechnology; Diagnosis; Treatment

Check for updates

OPEN ACCESS

\section{Introduction}

The coronavirus disease 2019 (COVID-19) outbreak began in Wuhan, Hubei Province, China. Severe acute respiratory syndrome coronavirus 2 (SARS-CoV-2) was recognized as the causative agent of this outbreak. The virus spreads through soft tissues such as the mouth, eyes and nose [1]. The disease is caused by SARS-CoV-2, a single-stranded RNA virus with a small genome of 26-32 kb, ranging from $65-125 \mathrm{~nm}$ in diameter. Initially, Chinese researchers named the virus the 2019 novel coronavirus (2019-nCoV) [2]. The disease spread rapidly throughout China within a month, and according to theWorld Health Organization (WHO), COVID-19 has currently spread throughout 212 countries, areas, and regions worldwide [3]. On a clinical basis, the two key requirements to controlling the COVID-19 pandemic are first to overcome the infection rate, and second to decrease the death rate [4]. Currently, no approved drugs or vaccines are available to treat or prevent COVID-19. However, newly developed vaccines are in the first stages of clinical trials. Current available therapies, including nonspecific antivirals, antibiotics to treat secondary bacterial infections and sepsis, and corticosteroids to reduce inflammation, fail in treating the severe disease in which the hallmark is the cytokine storm induced by
COVID-19 in the lungs. This is visible as inflammatory lesions with ground glass opacity on computed tomography (CT) scans. Suitable therapeutics for treating the new COVID-19 disease are in urgent demand [4]. The viral infection causes a novel respiratory disease (SARS), with a prolonged deadly nature. Currently available drugs have been successful in moderately handling the symptoms, but no specific treatment or therapeutics have been defined. Deterring the outbreak early is important for protection among all states. Various forthcoming drugs that are thought to be directly applicable, as well as new therapeutic drugs being developed, include viral entry inhibitors, replication inhibitors, protease inhibitors, heterocyclic antivirals, nanodrug delivery systems, and biological therapeutics [5].

Nanoparticles are small but have a large surface-tovolume ratio, which gives them phenomenal, unique features. Because of these features, nanoparticles have been used in the fields of biotechnology, medicine, drug delivery, sensors, and DNA labeling and are treated as a bridge between bulk materials [6]. Nano approaches have been used extensively to improve antiviral drug delivery and efficacy, particularly with nucleoside analogs in conjunction with delivery systems, which have potential applications against drug-resistant human 
immunodeficiency virus infections [5,7]. The many available nano delivery systems can be used with newly developed drug formulations to efficiently deliver the drugs with faster therapeutic indices for COVID-19. However, this work remains in progress, and no nano drugs are currently available to treat COVID-19. Nanotechnology also plays a role in diagnosing COVID-19. In this paper, we discuss the role of nanotechnology in diagnosing and potentially treating COVID-19.

\section{Role of nanotechnology in diagnosing COVID-19}

The COVID-19 outbreak put global pressure on modern societies, particularly the infrastructure related to health care. Therefore, diagnostic tests unique to this infection are urgently needed to confirm positive cases, screen patients and perform viral surveillance. Diagnostics may play an important role in containing COVID-19, allowing rapid implementation of control measures that restrict the spread by detecting and isolating cases and through contact tracing (i.e., detection of people who may have come into contact with an infected patient). Opacity on CT scans of the lungs of COVID-19 patients differs from that of patients with healthy lungs: COVID-19 lungs are denser, more profuse and concomitant [8]. Currently COVID-19 is diagnosed via reverse transcription (RT)-PCR and screened via CT scans, but each technique has drawbacks. Molecular methods can diagnose diseases more accurately than can syndromic testing or CT scans because they can track and classify different pathogens [9]. Nanotechnology brings new opportunities for producing inexpensive and scalable detection methods, safe personal safety equipment and new successful medications. Nanosensors are now a reality and can detect very low bacterial and viral concentrations and thus alert clinicians even before symptoms have shown in patients with very low viral loads. According to the joint WHO taskforce and China, from the end of December 2019 to mid February 2020, 104 SARS-CoV-2 viral strains were isolated and sequenced with Illumina and Oxford nanopore sequencing [10]. Illumina sequencing is a sequence-by-synthesis process using solid-phase bridge amplifications, whereas nanopore sequencing involves translocating a DNA molecule through a protein pore and evaluating subsequent changes in voltage to determine the sequence. Research and technology production and deployment are our best weapons in the fight against COVID-19, and nanotechnology instruments can be modified to diagnose, treat and prevent the disease [11]. Different nano-based strategies, which have advantages over molecular methods, have been developed to diagnose COVID-19. The currently developed methods are discussed below.

Reverse transcription loop-mediated isothermal amplification (RT-LAMP) coupled with a nanoparticlesbased biosensor (NBS) assay

COVID-19 is a highly lethal respiratory disease that is rapidly spreading and has caused international anxiety. Currently, it is diagnosed by detecting the SARS-CoV-2 nucleic acid via real-time RT-PCR [12]. In addition to this method, other gene amplification methods have been used, but their main limitations are that they are complex, require skilled personnel, and are time-consuming. To avoid these limitations, Yang et al. developed an RT-LAMP assay to detect triple genes to rapidly diagnose SARS-CoV-2. In 2003, this method was used to test for the SARS coronavirus and was considered quick and easy [13].

An RT-LAMP NBS assay has since been developed to rapidly and accurately diagnose COVID-19 [14]. This RT-LAMP NBS assay merges LAMP amplification, reverse transcription, and multiplex analysis with nanoparticle-based biosensors to facilitate diagnosing COVID-19 in a one-step, single-tube reaction. This method takes 30 minutes for nucleic acid

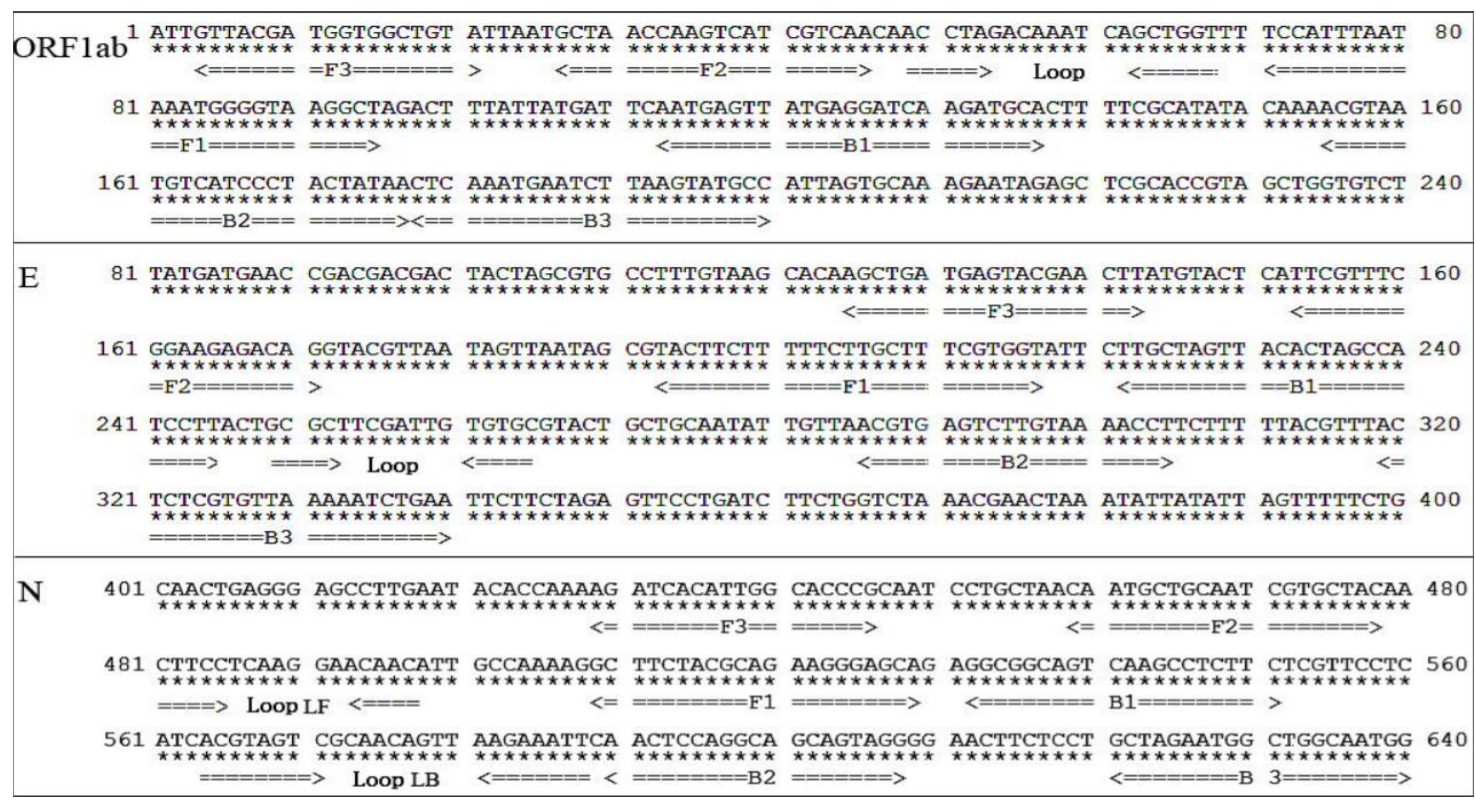


amplification at a constant $60-65^{\circ} \mathrm{C}$ using different primers to detect the N, E and ORF1ab genes. Approximately ten sets of primers have been designed per gene as shown in figure 1 .

The LAMP technique is very precise because it identifies the target sequence via six or eight different regions, directly using the RNA as a template [8]. The detection results can be seen macroscopically through the color change. These techniques include amplification of recombinase polymerase, helicase-based amplification, and loop-mediated isothermal amplification. Several academic labs have developed and clinically tested RT-LAMP tests for SARS-CoV-2 $[8,13]$. A one-step, one-tube RT-LAMP-NBS assay was developed to diagnose COVID-19. This method is advantageous because it is simple to operate and only requires basic, inexpensive equipment (e.g., a water bath or heating block) to maintain a constant temperature $\left(63^{\circ} \mathrm{C}\right)$ for $30-40$ minutes [15]. Studies have shown that compared with previously developed COVID-19 RT-LAMP assays, NBS, a simple and easy-to-use tool, can visually and objectively indicate the RT-LAMP results, eliminating the need for complicated processes (e.g., electrophoresis), special reagents (e.g., $\mathrm{pH}$ indicators) and expensive instruments (e.g., real-time PCR) [15,16]. Two target sequences are simultaneously amplified in an isothermal reaction and detected in the test stage. Future studies should determine the basic COVID-19 RT-LAMP theory, optimize the reaction parameters (e.g., amplification temperature), and demonstrate its feasibility. The specificity of the COVID-19 RTLAMP-NBS test was examined by detecting templates derived from various pathogens, including viruses, fungi and bacteria [14]. Mechanistic Description of the COVID-19 RT-LAMP-NBS assay shown in figure 2 .

\section{Point-of-care testing}

Point-of-care tests are used to treat patients without sending samples to centralized laboratories, yielding results without requiring a laboratory network to identify infected patients. Lateral-flow antigen detection for SARS-CoV-2 is a care point being considered for diagnosing COVID-19 [17]. Commercial lateral flow assays contain a paper-like membrane strip coated with two lines: one contains gold nanoparticleantibody conjugates; the other catches the antibodies. Patients' samples (e.g., blood and urine) are collected on the membrane, and capillary action pulls the protein across the line. When the first line moves, the antigens bind to the nanoparticle-antibody gold conjugate, and the complex flows across the membrane. The caught antibodies immobilize the complex when they enter the second side where the red or blue side is visible. Individual gold nanoparticles are red, and the coupled plasmon bands cause the solution containing the

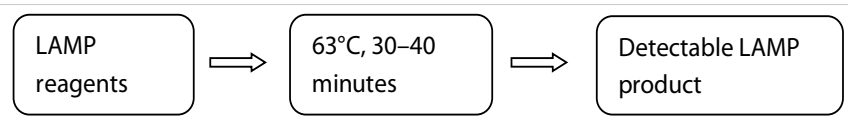

Figure 2: Three steps method of COVID-19 RT-LAMP-NBS assay. clustered gold nanoparticles to turn blue [18]. In academic laboratories, many platforms, such as electrochemical sensors, paper-based systems and surface-enhanced Raman scattering-based systems, are being developed.

\section{Optical biosensor nanotechnology}

A new system based on optical biosensor nanotechnology will enable detecting the coronavirus in approximately 30 minutes directly from patients' samples without requiring centralized laboratory tests. This new technology could easily determine whether a patient is infected with the coronavirus or the influenza virus. This project will potentially be used for more than the present pandemic and for treating humans. The new biosensor tool will also be used to analyze various forms of coronavirus present in reservoir animals, such as bats, to detect and track the potential evolution of these viruses and prevent future outbreaks in humans [19].

\section{Nanopore target sequencing (NTS)}

The NTS method simultaneously detects SARS-CoV-2 along with ten other respiratory viruses within only 6-10 hours. It is appropriate for contemporary diagnosing of COVID-19; however, the framework can be expanded to diagnose other viruses and pathogens. NTS is based on amplification of 11 SARS-CoV-2 virulence-related and unique gene fragments (e.g., orf1ab) using an internal primary panel followed by sequencing of the amplified fragment on a nanopore platform [20]. This project uses a nanopore platform for sequencing, which can sequence long nucleic acid fragments and simultaneously analyze the data output in real time. This allows confirming SARS-CoV-2 infections within minutes of sequencing by mapping the sequence reads to the SARS$\mathrm{CoV}-2$ genome and analyzing the identity, validity and read number of the output sequence. Based on the virulence region (genome 21,563-29,674 bp; NC 045512.2) encoding S (1273 amino acids; AA), ORF3a (275 AA), E (75 AA), M (222 AA), ORF6 (66 AA), ORF7a (121 AA), ORF8 (121 AA), N (419 $\mathrm{AA}$ ), and ORF10 (38 AA) proteins to detect pivotal SARSCoV-2 virulence genes. The NTS is conducted on one MinION sequencer chip for all test samples, and the sequence data are analyzed using an in-house bioinformatics pipeline at regular intervals. To increase plasmid concentrations, all highidentity reads were determined by mapping output reads on the SARS-CoV-2 genome. As a standard for qPCR, NTS cannot determine whether a sample is positive for infection by testing only one or two sites; findings from all target regions should be considered [21,22].

\section{Role of nanotechnology in treatment}

The rapid spread of COVID-19 requires effective therapeutic strategies to combat this deadly pneumonia. However, no specific drugs have been approved to treat COVID-19 [23]. Based on the experience of clinicians as well as pharmacological mechanisms, several immunomodulatory and antiviral 
medications have been suggested as potential investigational therapies, and many are now in clinical trials. These immunomodulatory and antiviral drugs include remdesivir, oseltamivir, favipiravir, darunavir/cobicistat, mycophenolate, tocilizumab, interferon, teicoplanin, convalescent plasma, and ribavirin, etc [24]. However, on 28 March 2020, the Food and Drug Administration issued an emergency use authorization for an oral formulation of hydroxychloroquine sulfate and chloroquine phosphate to treat COVID-19 [25, 26]. According to the latest literature, guidelines published in Belgium, which also included recommendations from the Netherlands, Switzerland, France and Italy, recommend remdesivir, ritonavir/lopinavir, chloroquine or hydroxychloroquine and tocilizumab in addition to supportive care, including mechanical ventilation and oxygen [27]. Favipiravir, an antiviral agent approved in China and Japan for treating influenza, is under investigational use against COVID-19 [28].

In addition to these proposed drugs, Chinese medicines play important roles in treating COVID-19. The Chinese Academy of Sciences along with the Shanghai Institute of Materia Medica suggested that SARS-CoV-2 could be prevented using Shuanghuanglian oral liquid [29,30]. This liquid contains compounds such as baicalin, chlorogenic acid and forsythin and shows antiviral and antibacterial properties [31]. Currently no vaccines have been approved for COVID-19; however, some newly developed vaccines are in clinical trials.

Nanotechnology can significantly contribute to treating COVID-19. Different nanoparticles have antiviral and antibacterial activities [11]. These nanoparticles can be used to reduce disease severity and risk. To date, no nano-based drugs are available to treat COVID-19. However, work is in progress, and nano-based drugs will hopefully be available in the near future. For example, zinc is a nanoparticle that may have important uses against COVID-19 [31].

\section{Can maintaining an optimal zinc balance enhance host resistance?}

Zinc nanoparticles can increase host resistance. Zinc has strong antiviral effects against rhinoviruses, infections with common cold viruses, and influenza viruses. Zinc could also be used to treat COVID-19 infections and reduce the disease burden and intensity [32]. Zinc nanoparticles reduce influenza viral replication in vitro, and zinc oxide nanoparticles show promise against H1N1 influenza viral infections. Zinc also inhibits replication of the SARS coronavirus [33,34]. Zinc salts inhibit respiratory syncytial viral replication and reduce hepatitis $\mathrm{C}$ viral replication. Zinc supplementation in children has significantly reduced the prevalence of pneumonia in developing countries. The antiviral mechanism of zinc remains unclear, but it may inhibit the virus from binding to the mucosa and subsequently replicating. In vitro studies have shown that zinc exerts antiviral effects by inducing generation of antiviral interferon alpha or gamma and by suppressing inflammatory reactions [31].
Zinc also influences immune cell functions and helps activate enzymes involved in different cellular functions [35]. Recent literature suggests that zinc deficiency leads to increased susceptibility to various bacterial and viral infections. In vivo studies have shown that zinc deficiency compromises B-cell development, which leads to low antibody production and macrophages with low phagocytic ability against parasites [36, 31]. Evidence shows that zinc exerts antiviral effects either by suppressing viral replication or by boosting the immune response. Data show that consuming approximately $50 \mathrm{mg}$ of zinc daily boosts the host immune system, thus making the host resistant to viral infection, and may provide an additional defense against COVID-19 infection to reduce the disease burden $[31,37,38]$. Mask Surface impregnated with ionic zinc oxide nanoparticles have strong antiviral and antibacterial efficacy. Incorporating zinc oxide nanoparticles into these materials can stabilize them and kill pathogens on contact $[39,40]$.

\section{Future prospects}

The world currently faces a global health emergency. The infection-to-mortality ratio from COVID-19 has crossed the limits, which makes it different from other viral infections. Physicians and scientists must work together to overcome the threat of SAR-CoV-2 and provide a strong base to block future pandemics. In the fight against COVID-19, research and technology development and deployment are our best weapons. Nanotechnology tools can be modified to treat, detect, and prevent the spread of COVID-19. Nanotechnology offers a unique set of tools and can substantially contribute to our understanding of viral diseases and toward the critical development of diagnostic and therapeutic platforms. Together, we can overcome this current pandemic to prevent and mitigate future viral outbreaks.

\section{Availability of data}

Data sharing not applicable to this article as no datasets were generated or analyzed during the current study.

\section{Authors' contribution}

AW and AK searched the databases and retrieved the relevant literature. $\mathrm{AB}$ arranged and compiled the format and handled the correspondence. AW, AA and MA selected the literature and finalized the manuscript. All authors read and approved the final manuscript.

\section{Acknowledgment}

The authors acknowledge Dr. Abdul Baset Bacha Khan University Charsadda and Mr. Misbahud Din for their valuable suggestions in improving this review. We thank Traci Raley, MS, ELS, for editing a draft of this manuscript.

\section{References}

1. Waris A, Khan AU, Ali M, Ali A, Baset A. COVID-19 outbreak: current scenario of Pakistan. New Microbes and New Infections. 2020.100681. 
2. Kaul D. An Overview of Coronaviruses including the SARS-2 Coronavirus-Molecular Biology, Epidemiology and Clinical Implications. Current Medicine Research and Practice, 2020.

PubMed: https://www.ncbi.nlm.nih.gov/pubmed/32363221

3. Curley M, Thomas N. Human security and public health in Southeast Asia: the SARS outbreak. Australian J Int Affairs, 2004. 58: 17-32.

4. Kumar S, Rathi B. Coronavirus Disease COVID-19: A New Threat to Public Health. Curr Top Med Chem. 2020; 20(8):599-600. PubMed: https://www.ncbi.nlm.nih.gov/pubmed/32133964

5. Agarwal HK, Chhikara BS, Doncel GF, Parang K. Synthesis and anti-HIV activities of unsymmetrical long chain dicarboxylate esters of dinucleoside reverse transcriptase inhibitors. Bioorganic \&amp; medicinal chemistry letters. 2017; 27: 1934-1937.

PubMed: https://www.ncbi.nlm.nih.gov/pubmed/28351588

6. Gnanasangeetha D, SaralaThambavani D. One pot synthesis of zinc oxide nanoparticles via chemical and green method. Res J Material Sci. 2013; 2320: 6055.

7. Chhikara BS. Corona virus SARS-CoV-2 disease COVID-19: Infection, prevention andclinical advances of the prospective chemical drug therapeutics. Chemical Biology Letters, 2020. 7: 63-72.

8. Chotiwan N, Brewster CD, Magalhaes T, Weger-Lucarelli J, Duggal NK, et al. Rapid and specific detection of Asian-and African-lineage Zika viruses. Science translational medicine. 2017; 9: eaag0538. PubMed: https://pubmed.ncbi.nlm.nih.gov/28469032

9. Yang W, Dang X. Rapid Detection of SARS-CoV-2 Using Reverse Transcription RT-LAMP Method. medRxiv. 2020.

10. Lu R, Zhao X, Li J, Niu P, Yang B, et al. Genomic characterisation and epidemiology of 2019 novel coronavirus: implications for virus origins and receptor binding. The Lancet. 2020; 395: 565-574.

11. Chan WCW. Nano Research for COVID-19. ACS Nano. 2020; 14 3719-3720.

PubMed: https://pubmed.ncbi.nlm.nih.gov/32227916/

12. Law S, Leung AW, Xu C. Severe Acute Respiratory Syndrome (SARS) and Coronavirus disease-2019 (COVID-19): From Causes to Preventions in Hong Kong. International Journal of Infectious Diseases. 2020.

PubMed: https://pubmed.ncbi.nlm.nih.gov/32251790

13. Yang W, Dang X, Wang Q, Xu M, Zhao Q, et al. Rapid Detection of SARS-CoV-2 Using Reverse transcription RT-LAMP method. Med Rxiv. 2020.

14. Zhu X, Wang X, Han L, Chen T, Wang L, et al. Reverse transcription loop-mediated isothermal amplification combined with nanoparticlesbased biosensor for diagnosis of COVID-19. medRxiv. 2020.

15. Yu L, Wu S, Hao X, Li X, Liu X, et al. Rapid colorimetric detection of COVID-19 coronavirus using a reverse tran-scriptional loop-mediated isothermal amplification (RT-LAMP) diagnostic plat-form: iLACO. medRxiv. 2020

16. Lamb LE, Bartolone SN, Ward E, Chancellor MB. Rapid Detection of Novel Coronavirus (COVID-19) by Reverse Transcription-LoopMediated Isothermal Amplification. medRxiv. 2020.

17. Xiang J, Yan M, Li H, Liu T, Lin C, et al. Evaluation of Enzyme-Linked Immunoassay and Colloidal Gold-Immunochromatographic Assay Kit for Detection of Novel Coronavirus (SARS-Cov-2) Causing an Outbreak of Pneumonia (COVID-19). medRxiv. 2020.

18. Udugama B, Kadhiresan $P$, Kozlowski HN, Malekjahani A, Osborne M, et al. Diagnosing COVID-19: The Disease and Tools for Detection. ACS nano. 2020.

19. Zhu X, Wang X, Han L, Chen T, Wang L, et al. Reverse transcription loop-mediated isothermal amplification combined with nanoparticlesbased biosensor for diagnosis of COVID-19. medRxiv. 2020.

20. Wang M, Fu A, Hu B, Tong Y, Liu R, et al. Nanopore target sequencing for accurate and comprehensive detection of SARS-CoV-2 and other respiratory viruses. medRxiv. 2020.

21. Liu R, Fu A, Deng Z, Li Y, Liu T. Promising methods for detection of novel coronavirus SARS-CoV-2. View. 2020; 1.

22. Yu L, Tong Y, Shen G, Fu A, Lai $Y$, et al. Immunodepletion with Hypoxemia: A Potential High Risk Subtype of Coronavirus Disease 2019. medRxiv. 2020.

23. Peeri NC, Shrestha N, Rahman MS, Zaki R, Tan Z, et al. The SARS MERS and novel coronavirus (COVID-19) epidemics, the newest and biggest global health threats: what lessons have we learned? Int $J$ Epidemiol. 2020 Feb 22.

PubMed: https://www.ncbi.nlm.nih.gov/pubmed/32086938

24. Ziaie S, Koucheck M, Miri M, Salarian S, Shojaei S, et al. Review of therapeutic agents for the treatment of COVID-19. J Cellular Molecular Anesthe. 2020; 5: 32-36.

25. Song Z, Hu Y, Zheng S, Yang L, Zhao R. Hospital pharmacists' pharmaceutical care for hospitalized patients with COVID-19: Recommendations and guidance from clinical experience. Res Social Adm Pharm. 2020.

PubMed: https://www.ncbi.nlm.nih.gov/pmc/articles/PMC7129111/

26. Tobaiqy M, Qashqary M, Al-Dahery S, Mujallad A, Hershan AA, et al. Therapeutic Management of COVID-19 Patients: A systematic review. Infection Prevention in Practice. 2020: 100061.

27. Donders F, Lonnée-Hoffmann R, Tsiakalos A, Mendling W, Martinez de Oliveira J, et al. COVID I. ISIDOG Recommendations Concerning COVID-19 and Pregnancy. Diagnostics. 2020; 10: 243.

PubMed: https://www.ncbi.nlm.nih.gov/pubmed/32338645

28. Sanders JM, Monogue ML, Jodlowski TZ, Cutrell JB. Pharmacologic treatments for coronavirus disease 2019 (COVID-19): a review. JAMA 2020

PubMed: https://www.ncbi.nlm.nih.gov/pubmed/32282022

29. Wang LS, Wang YR, Ye DW, Liu QQ. A review of the 2019 Novel Coronavirus (COVID-19) based on current evidence. Int J Antimicrob Agents. 2020: 105948.

PubMed: https://www.ncbi.nlm.nih.gov/pmc/articles/PMC7156162

30. Kaura KK, Allahbadiab G, Singhc M. A comprehensive review on epidemiology, aetiopathogenesis, diagnosis and treatment of the novel coronavirus syndrome-COVID-19. 2020.

31. Dong L, Hu S, Gao J. Discovering drugs to treat coronavirus disease 2019 (COVID-19). Drug discoveries \& therapeutics. 2020; 14: 58-60. PubMed: https://www.ncbi.nlm.nih.gov/pubmed/32147628

32. Razzaque M. COVID-19 Pandemic: Can Maintaining Optimal Zinc Balance Enhance Host Resistance?

33. Eby GA. Zinc ion availability--the determinant of efficacy in zinc lozenge treatment of common colds. The Journal of antimicrobial chemotherapy. 199; 40: 483-493.

PubMed: https://www.ncbi.nlm.nih.gov/pubmed/9372416

34. Read SA, Obeid S, Ahlenstiel C, Ahlenstiel G. The role of zinc in antiviral immunity. Adv Nutr. 2019; 10: 696-710.

PubMed: https://www.ncbi.nlm.nih.gov/pubmed/31305906

35. Eby III GA. Zinc lozenges as cure for the common cold-a review and hypothesis. Med Hypotheses. 2010; 74: 482-492.

PubMed: https://www.ncbi.nlm.nih.gov/pubmed/19906491

36. Field CJ, Johnson IR, Schley PD. Nutrients and their role in host 
resistance to infection. J leukoc Biol. 2002; 71: 16-32.

PubMed: https://pubmed.ncbi.nlm.nih.gov/11781377

37. Prasad AS. Zinc: mechanisms of host defense. J Nutr. 2007; 137 1345-1349.

PubMed: https://www.ncbi.nlm.nih.gov/pubmed/17449604

38. Overbeck S, Uciechowski P, Ackland ML, Ford D, Rink L. Intracellular zinc homeostasis in leukocyte subsets is regulated by different expression of zinc exporters ZnT-1 to ZnT-9, J Leukoc Biol. 2008; 83: 368-380.

PubMed: https://www.ncbi.nlm.nih.gov/pubmed/17971500
39. Lambert SA, Jolma A, Campitelli LF, Das PK, Yin Y, et al. The Human ranscription Factors. Cell. 2018; 172: 650665.

PubMed: https://www.ncbi.nlm.nih.gov/pubmed/30290144

40. Wimalawansa SJ. Global epidemic of coronavirus-covid-19: what can we do to minimize risks. European J Biomed. 2020; 7: 432-438.

41. Król A, Pomastowski P, Rafińska K, Railean-Plugaru V, Buszewski B. Zinc oxide nanoparticles: Synthesis, antiseptic activity and toxicity mechanism. Advances in colloid and interface science. 2017; 249: 37-52.

PubMed: https://www.ncbi.nlm.nih.gov/pubmed/28923702 